\title{
Cyclic-Averaging for High-Speed Analysis of Resonant Converters
}

\author{
Martin P. Foster, H. Isaac Sewell, Chris M. Bingham, Member, IEEE, David A. Stone, Dirk Hente, and Dave Howe
}

\begin{abstract}
The paper describes the development and application of a cyclic-averaging technique for the rapid analysis of high-order resonant power converters. To provide a focus to the paper, particular emphasis is given to a 3rd-order LCC voltage output converter topology. The proposed methodology predicts steady-state voltages and currents throughout the circuit, and provides estimates of the stresses on the resonant circuit components. State-space simulations and experimental results from a $350 \mathrm{~V}$-input/150 V-output converter are used to demonstrate a prediction accuracy comparable with time-domain integration-based techniques is achievable, while requiring only $1 / 10,000$ th of the computation time. In addition, a comparison with Spice simulation results shows that cyclic averaging provides commensurate predictions of voltage and current stresses on the resonant circuit components. Issues arising from the stray capacitance associated with the resonant inductor, and the corresponding sensitivity of the predicted output voltage, are also considered.
\end{abstract} tion.

Index Terms-Modelling, resonant power conversion, simula-

\section{INTRODUCTION}

$\mathbf{R}$ ESONANT power converters offer a higher efficiency and reduced size compared with traditional switched-mode counterparts. Thus, there is significant interest in high-order resonant systems, such as that shown in Fig. 1, particularly in the consumer product industry, to satisfy the requirement for smaller power supplies for compact electronic equipment. However, the increased circuit complexity makes it more difficult to accurately predict the performance of the converters during an iterative design process, due to the protracted simulation times required for component-based simulation packages, such as Spice. Computationally efficient algorithms are, therefore, sought to accurately predict current and voltage waveforms throughout such converters, so as to facilitate their design.

Utilizing state-variable models, and exploiting the periodic behavior of resonant converters in the steady-state, cyclic-averaging techniques will be shown to provide an attractive alternative to traditional 'integration-based' and harmonic analysis methods for converter performance evaluation, and component stress prediction.

Manuscript received February 8, 2002; revised March 3, 2003. Recommended by Associate Editor K. Smedley.

M. P. Foster, H. I. Sewell, C. M. Bringham, D. A. Stone, and D. Howe are with the Department of Electronic and Electrical Engineering, University of Sheffield, Sheffield, S1 3JD U.K. (e-mail: c.bingham@ sheffield.ac.uk).

D. Hente is with the Forschlungslaboratorien, Philips GmbH, Aachen, Germany.

Digital Object Identifier 10.1109/TPEL.2003.813763

\section{Modelling CyCLIC BeHAVIOUR}

The proposed approach is considered to be a variant of Floquet-based techniques, [1], whereby averaged, steady-state values of state-variables are determined. Due to the multiple operating modes of resonant converters, Fig. 2, "classical" steady-state operating points cannot be determined due to the continuous switching nature of the input voltage. However, by considering converter operation to be periodic, then

$$
x(t)=x(t+n T)
$$

where the state vector, $x$, describing converter operation at time $t$ is equal to the state vector at time $t+n T, T$ being the period of the applied input voltage and $n$ an integer. The resulting performance of the converter can then be obtained from analytical equations without the need for integration. The method can, thereby, provide steady-state performance predictions extremely rapidly.

In the steady-state, each period of a cycle can be decomposed into multiple operating modes, each dependent on the state of the input voltage and the rectifier input/output voltages and currents. Fig. 2 shows typical steady-state voltage and current waveforms for the resonant converter circuit shown in Fig. 1, together with the sub-division of the cycle into operating modes, $\mathrm{M}_{1} \rightarrow \mathrm{M}_{6}$.

In general, power converters operating in a cyclic-mode can be modeled by a system of piecewise linear (state-space) equations which describe the converter in each operating mode during the cycle; i.e.

$$
\dot{x}_{i}=A_{i} x_{i}+B_{i}
$$

where $x_{i}$ is the state vector, $A_{i}$ is the dynamical matrix and $B_{i}$ is the input vector, in the $i^{\text {th }}$ operating mode of the converter. Thus, they consist of a linear combination of circuit voltages and currents. For the $i^{t h}$ mode, (2) can be solved analytically

$$
\begin{aligned}
x_{i}(t) & =e^{A_{i} t} x_{i}\left(t_{0}\right)+\int_{t_{0}}^{t} e^{A_{i}(t-\tau)} B_{i} d \tau \\
& =\Phi_{i} x_{i}\left(t_{0}\right)+\Gamma_{i}
\end{aligned}
$$

where $\Phi_{i}=\Phi\left(t, t_{0}\right)=e^{A_{i} t}, \Gamma_{i}=\int_{t_{0}}^{t} e^{A_{i}(t-\tau)} B_{i} d \tau$, and $x_{i}\left(t_{0}\right)$ are the initial conditions for the mode. If the time during which the circuit operates in the $i^{t h}$ mode is $d_{i} T$, where $d_{i}$ is the associated duty-cycle, the complete solution for the dynamics of the converter can be obtained by employing the state vector at time $d_{i} T$ as the initial condition for the subsequent dynamics of the $(i+1)^{t h}$ mode. Nevertheless, the solution of (3) is complicated by the need to evaluate the integral, which is a major contribution to the computational overhead when analyzing the 


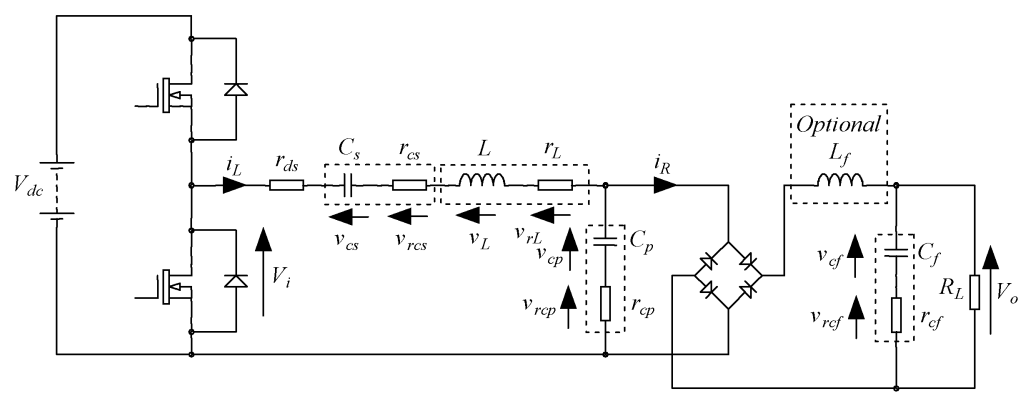

Fig. 1. Third-order LCC resonant converter (including parasitic elements).

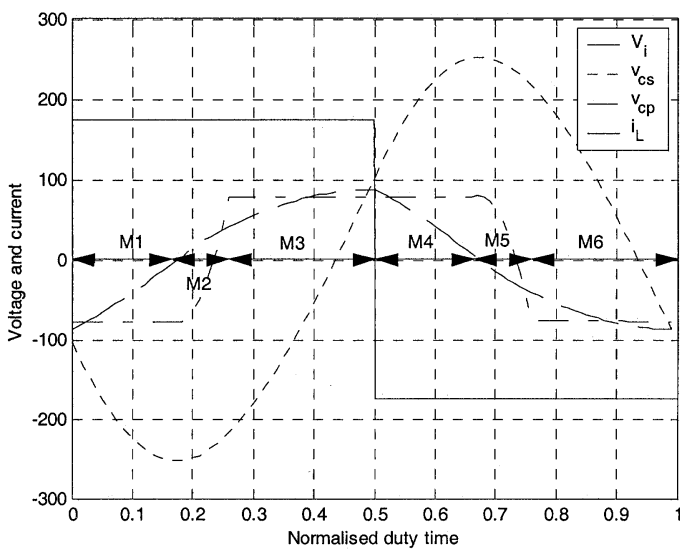

Fig. 2. Voltages and currents of a third order voltage output LCC resonant converter.

system in this manner. However, by combining $A_{i}$ and $B_{i}$ to form an augmented system matrix, (4), the integration overhead can be eliminated at the expense of obtaining only the cyclic steady-state description

or

$$
\frac{d}{d t}\left(\begin{array}{c|c}
x_{i}(t) \\
\hline 1
\end{array}\right)=\left(\begin{array}{c|c}
A_{i} & B_{i} \\
\hline 0 & 0
\end{array}\right)\left(\begin{array}{c}
x_{i}(t) \\
\hline 1
\end{array}\right)
$$

$$
\frac{d}{d t} \hat{x}_{i}(t)=\hat{A}_{i} \hat{x}_{i}(t)
$$

Consider the solution of the state vector, (4), at the transition time between modes $1 \& 2$, $\left(\right.$ denoted $\left.t_{1}\right)$

$$
\hat{x}_{1}\left(t_{1}\right)=e^{\hat{A}_{1} d_{1} T} \hat{x}_{1}\left(t_{0}\right)=\hat{\Phi}_{1} \hat{x}_{1}\left(t_{0}\right)
$$

where $\hat{x}_{1}\left(t_{0}\right)$ are the initial conditions of the cyclic mode, and $\hat{\Phi}_{1}=\hat{\Phi}\left(t_{1}, t_{0}\right)=e^{\hat{A}_{1} d_{1} T}$. In a similar manner, the solution of the state-vector at the end of mode 2 can be obtained from

$$
\hat{x}_{2}\left(t_{2}\right)=e^{\hat{A}_{2} d_{2} T} \hat{x}_{2}\left(t_{0}\right)=\hat{\Phi}_{2} \hat{\Phi}_{1} \hat{x}_{1}\left(t_{0}\right) .
$$

In general, for a system with $m$-modes, the state-vector at the end of the $m^{t h}$ mode is

$$
\hat{x}_{m}\left(t_{m}\right)=\hat{\Phi}_{m} \hat{\Phi}_{m-1} \ldots \hat{\Phi}_{1} \hat{x}_{1}\left(t_{0}\right)=\hat{\Phi}_{t o t} \hat{x}_{1}\left(t_{0}\right)=\hat{x}_{1}\left(t_{0}\right)
$$

where $\hat{x}\left(t_{m}\right)$ is the state-vector at time $t_{m}, \hat{\Phi}_{\text {tot }}=$ $\prod_{i=m, m-1, \ldots}^{1} \hat{\Phi}_{i}$ and $\hat{\Phi}_{i}=\left(\begin{array}{c|c}\Phi_{i} & \Gamma_{i} \\ \hline 0 & 1\end{array}\right)$ Due to the peri- odic nature of the system, the initial condition, $x_{p e r}\left(t_{0}\right)$, for operation in a cyclic mode is given by

$$
x_{p e r}\left(t_{0}\right)=\left(I^{n}-\Phi_{t o t}\right)^{-1} \Gamma_{t o t}
$$

where $\Phi_{t o t}=\Phi_{m} \Phi_{m-1} \cdots \Phi_{1}$ and

$$
\begin{aligned}
\Gamma_{t o t}=\left(\Phi_{m} \Phi_{m-1} \cdots \Phi_{2}\right) \Gamma_{1}+ & \left(\Phi_{m} \Phi_{m-1} \cdots \Phi_{3}\right) \\
\times & \Gamma_{2}+\cdots+\left(\Phi_{m}\right) \Gamma_{m-1}+\Gamma_{m} .
\end{aligned}
$$

From (7), the value of the state-variables at any particular instant in time, $t$, within a cyclic mode can be predicted by using knowledge of the initial conditions, $x_{p e r}\left(t_{0}\right)$, for a particular mode, $i$

$$
\begin{aligned}
\hat{x}(t)= & e^{\hat{A}_{i}\left(t-T \sum_{m=1}^{i-1} d_{m}\right)} \\
& \times\left(\prod_{n=i-1, i-2, \cdots}^{1} \hat{\Phi}_{n}\right) \hat{x}_{p e r}\left(t_{0}\right)
\end{aligned}
$$

where

$$
\hat{x}_{p e r}\left(t_{0}\right)=\left[\frac{x_{p e r}\left(t_{0}\right)}{1}\right]
$$

\section{Averaged State-VARIABLES}

In order to obtain the average steady-state output voltage of the converter, the mean values of the state-variables over a complete cycle are found from

$$
x_{a v}=\frac{1}{T} \int_{t_{0}}^{t_{0}+T} x(t) d(t) .
$$

Again, the requirement for integration impedes efficient analysis of the system. However, by augmenting the state-vector with $\dot{x}_{a v}(t)=(1 / T) x(t)$, the solution of the resulting system can be simplified. From [1], the dynamics of the converter during the $i^{\text {th }}$ mode of a cycle are given by

$$
\begin{aligned}
\dot{x}_{i} & =A_{i} x_{i}+B_{i} \\
\dot{x}_{i_{-} a v} & =\frac{d_{i}}{T} x_{i_{-} a v}
\end{aligned}
$$

with the resulting dynamic description

or

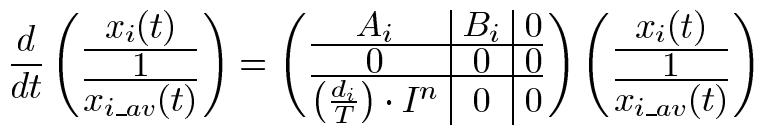

$$
\dot{z}_{i}(t)=\tilde{A}_{i} z_{i}(t)
$$


The initial conditions for the cyclic mode are now:

$$
z\left(t_{0}\right)=\left(\frac{\frac{x_{p e r}\left(t_{0}\right)}{1}}{0}\right)
$$

In a manner similar to that presented in the previous section, the averaged state-vector is obtained as follows

$$
\begin{aligned}
& z\left(t_{0}+T\right)=\tilde{\Phi}_{m} \tilde{\Phi}_{m-1} \cdots \tilde{\Phi}_{1} z\left(t_{0}\right) \\
& z\left(t_{0}+T\right)=\left(\frac{\frac{x_{p e r}\left(t_{0}\right)}{1}}{x_{a v}}\right)
\end{aligned}
$$

from which the average output voltages (or currents) of switching converters, together with the average voltages (or currents) across (or through) the reactive components in the circuit, $x_{a v}$, are determined.

\section{State-Space Model of LCC Voltage OUTPUT CONVERTER}

To demonstrate the attributes of cyclic averaging for the analysis of resonant power converters, a 3rd order LCC voltage output converter, is considered, Fig. 1. The optional filter inductor, $L_{f}$ is often included for use in high-power converters and those which require a low output current ripple, and facilitates a reduction in the size of the output filter capacitor. However, to limit the physical size of the converter, and to reduce cost, the filter inductor is usually omitted. This does, however, significantly complicate the analysis since the voltage across the parallel resonant capacitor is clamped at the output voltage when the rectifier conducts, and cannot be considered to be sinusoidal (as in a FMA analysis). The treatment presented in the paper is solely concerned with the converter operating without a filter inductor.

To obtain the state-variable equations that describe the converter operation (omitting $L_{f}$ ), the circuit is first partitioned into two sub-systems, [2]. The filter capacitor and load are represented by a 'slow' sub-system and the resonant circuit by a 'fast' sub-system; the interaction between the two being through a rectifier coupling equation. The differential equations describing the fast sub-system are

$$
\begin{aligned}
& \frac{d v_{C p}}{d t}=\frac{i_{L}-i_{R}}{C_{p}}, \quad \frac{d v_{C s}}{d t}=\frac{i_{L}}{C_{p}} \\
& \frac{d i_{L}}{d t}=\frac{V_{i}-v_{C s}-v_{C p}-i_{L}\left(r_{d s}+r_{C s}+r_{L}+r_{c p}\right)+i_{R} r_{C p}}{L}
\end{aligned}
$$

and, similarly, for the slow sub-system

$$
\frac{d v_{C f}}{d t}=\frac{R_{L}}{C_{f}\left(R_{L}+r_{C f}\right)}\left|i_{R}\right|-\frac{v_{C f}}{C_{f}\left(R_{L}+r_{C f}\right)} .
$$

The coupling equation is obtained by equating the voltages across both sides of the rectifier; viz:

$v_{C p}+v_{r c p}=\operatorname{sgn}\left(i_{L}\right)\left(v_{o}+2 V_{d}\right)=\operatorname{sgn}\left(i_{L}\right)\left(v_{C f}+v_{r c f}+2 V_{d}\right)$

where $V_{d}$ is the rectifier voltage drop. Assuming the parasitic resistance of each capacitor has a negligible effect, (17) reduces to

$$
v_{C p}=\operatorname{sgn}\left(i_{L}\right)\left(v_{C f}+2 V_{d}\right)
$$

from which the dynamic equation describing the parallel capacitor voltage is obtained as

$$
\frac{d v_{C p}}{d t}=\operatorname{sgn}\left(i_{L}\right)\left(\frac{d v_{C f}}{d t}\right) .
$$

Substituting (15) and (16) into (19) for the appropriate capacitor currents, and solving for the rectifier current, $i_{R}$, yields

$$
i_{R}=\frac{C_{f}}{C_{f}+C_{p}} i_{L}+\operatorname{sgn}\left(i_{L}\right) \frac{C_{p}}{R_{L}\left(C_{f}+C_{p}\right)} v_{C f} .
$$

The combined state-space model and coupling equation are then given by (21) and (22), shown at the bottom of the page. Note: For use in signal-conservative simulation packages, e.g., Simulink, modifications to the coupling equation are required to ensure the rectifier commutates correctly, see [3].

\section{Derivation of Cyclic Model}

Analysis of the behavior of the third-order voltage output LCC converter, Fig. 1, shows that for switching frequencies above, and in the region of, the resonant frequency, six operating modes are present in each periodic cycle, with respect

$$
\begin{aligned}
{\left[\begin{array}{c}
\dot{v}_{C p} \\
\dot{v}_{C s} \\
\dot{i}_{L} \\
\dot{v}_{C f}
\end{array}\right]=} & {\left[\begin{array}{cccc}
0 & 0 & \frac{1}{C_{p}} & 0 \\
0 & 0 & \frac{1}{C_{s}} & 0 \\
-\frac{1}{L} & -\frac{1}{L} & -\frac{r_{d s}+r_{C s}+r_{C p}+r_{L}}{L} & 0 \\
0 & 0 & 0 & -\frac{1}{C_{f}\left(R_{L}+r_{C f}\right)}
\end{array}\right] } \\
& \times\left[\begin{array}{c}
v_{C p} \\
v_{C s} \\
i_{L} \\
v_{C f}
\end{array}\right]+\left[\begin{array}{c}
-\frac{i_{R}}{C_{p}} \\
0 \\
\frac{V_{i}}{L}+\frac{i_{R} r_{C p}}{L} \\
\frac{\left|i_{R}\right| R_{L}}{C_{f}\left(R_{L}+r_{C f}\right)}
\end{array}\right] \\
v_{o}= & \frac{R_{L}}{R_{L}+r_{C f}} v_{C f}+\frac{R_{L} r_{C f}}{R_{L}+r_{C f}}\left|i_{R}\right| \\
i_{R}= & \left\{\begin{array}{cc}
\frac{C_{f}}{C_{f}+C_{p}} i_{L}+\operatorname{sgn}\left(i_{L}\right) \frac{C_{p}}{R_{L}\left(C_{f}+C_{p}\right)} v_{C f} & \left|v_{c p}\right|>v_{C f}+2 V_{d} \\
0 & \left|v_{c p}\right| \leq v_{C f}+2 V_{d}
\end{array}\right.
\end{aligned}
$$


to the polarity of the input voltage and the state of the rectifier current, Fig. 2. Although other mode sequences can occur, for operation well above resonance, these impart poor switch power-factor characteristics, and are not normally considered for design purposes.

The derivation of the piece-wise linear equations required for cyclic averaging is complicated by the requirement for calculating the magnitude of the rectifier current, $i_{R}$ (i.e. $\left|i_{R}\right|$ is a nonlinear function of $i_{L}$ and $v_{C f}$ ). However, a simplified expression for $i_{R}$ can be obtained by noting that the filter capacitor, $C_{f}$, is much larger in value than the parallel capacitor, $C_{p}$, for a 'ripple-free' output voltage. Hence, $v_{C f}$ in (22) can be neglected. Thus

$$
i_{R}=\left\{\begin{array}{cl}
\frac{C_{f}}{C_{f}+C_{p}} i_{L} & \left|v_{c p}\right|>v_{C f}+2 V_{d} \\
0 & \left|v_{c p}\right| \leq v_{C f}+2 V_{d}
\end{array}\right.
$$

the dynamic matrices, $A_{i}$, and the input matrices, $B_{i}$, are found by substitution of (23) into (21) and taking account of the polarity of the input voltage and the direction of the rectifier current. In particular, for operation in mode $1\left(V_{i}>0, i_{R}<0\right)$ as (24), shown at the bottom of the page, and the coupling equation is

$$
\begin{aligned}
i_{R} & =\frac{C_{f}}{C_{f}+C_{p}} i_{L} \\
\left|i_{R}\right| & =-\frac{C_{f}}{C_{f}+C_{p}} i_{L} .
\end{aligned}
$$

Similarly, the modal equations describing mode $2\left(V_{i}>0\right.$, $\left.i_{R}=0\right)$ are

$$
\begin{aligned}
A_{2} & =\left[\begin{array}{cccc}
0 & 0 & \frac{1}{C_{p}} & 0 \\
0 & 0 & \frac{1}{C_{s}} & 0 \\
-\frac{1}{L} & -\frac{1}{L} & -\frac{r_{d s}+r_{C s}+r_{C p}+r_{L}}{L} & 0 \\
0 & 0 & 0 & -\frac{1}{C_{f}\left(R_{L}+r_{C f}\right)}
\end{array}\right] \\
B_{2} & =\left[\begin{array}{c}
0 \\
0 \\
\frac{V_{i}}{L} \\
0
\end{array}\right]
\end{aligned}
$$

whilst for mode $3\left(V_{i}>0, i_{R}>0\right)$ as (27), shown at the bottom of the page.

Due to symmetry, the modal matrices for modes 4,5,6 are equivalent to those given previously, i.e.

$$
A_{4}=A_{3}, B_{4}=B_{5}=B_{6}=\left[\begin{array}{c}
0 \\
0 \\
-\frac{V_{i}}{L} \\
0
\end{array}\right], A_{5}=A_{2}, A_{6}=A_{1} .
$$

The time-periods associated with each mode are derived from an equivalent circuit based on a modified Rectifier-Compensated Fundamental Mode Analysis (RCFMA) [4] technique. The equivalent circuit models the combined nonlinear effects of the bridge rectifier and the output filter, by an equivalent resistance, $R_{e q}$, and capacitance, $C_{e q}$, Fig. 3 , is described by

$$
A_{1}=\left[\begin{array}{cccc}
0 & 0 & \frac{1}{C_{p}}-\frac{C_{f}}{C_{p}\left(C_{f}+C_{p}\right)} & 0 \\
0 & 0 & \frac{1}{C_{s}} & 0 \\
-\frac{1}{L} & -\frac{1}{L} & -\frac{r_{d s}+r_{C s}+r_{C p}+r_{L}}{L}+\frac{r_{c p} C_{f}}{L\left(C_{f}+C_{p}\right)} & 0 \\
0 & 0 & -\frac{R_{L}}{C_{f}\left(R_{L}+r_{c f}\right)} \cdot \frac{C_{f}}{C_{f}+C_{p}} & -\frac{1}{C_{f}\left(R_{L}+r_{c f}\right)}
\end{array}\right], \quad B_{1}=\left[\begin{array}{c}
0 \\
0 \\
\frac{V_{i}}{L} \\
0
\end{array}\right]
$$

$$
A_{3}=\left[\begin{array}{cccc}
0 & 0 & \frac{1}{C_{p}}-\frac{C_{f}}{C_{p}\left(C_{f}+C_{p}\right)} & 0 \\
0 & 0 & \frac{1}{C_{s}} & 0 \\
-\frac{1}{L} & -\frac{1}{L} & -\frac{r_{d s}+r_{C s}+r_{C p}+r_{L}}{L}+\frac{r_{c p} C_{f}}{L\left(C_{f}+C_{p}\right)} & 0 \\
0 & 0 & \frac{R_{L}}{C_{f}\left(R_{L}+r_{c f}\right)} \cdot \frac{C_{f}}{C_{f}+C_{p}} & -\frac{1}{C_{f}\left(R_{L}+r_{c f}\right)}
\end{array}\right], \quad B_{3}=\left[\begin{array}{c}
0 \\
0 \\
\frac{V_{i}}{L} \\
0
\end{array}\right]
$$

$$
\begin{aligned}
V_{e q} & =\frac{2}{\pi} V_{i n} \\
r_{l} & =\frac{8}{\pi^{2}} R_{L} \\
R_{e q} & =\frac{8 R_{L}}{\pi^{2}\left(1+4 R_{L} f C_{P}\right)}+\frac{8 V_{\text {diode }}}{I_{\text {in }} \pi\left(1+4 R_{L} f C_{P}\right)}-\frac{64 f C_{P}\left(R_{L} I_{i n}+\pi V_{\text {diode }}\right)^{2}}{\pi^{2} I_{i n}^{2}\left(1+4 R_{L} f C_{P}\right)^{2}} \\
C_{e q} & =\frac{\pi C_{P}\left(4 f C_{p} R_{L}+1\right)^{2}}{4\left(4 f C_{p} R_{L}-1\right) \sqrt{f C_{P} R_{L}}+\pi\left(4 f C_{p} R_{L}+1\right)^{2}-\left(4 f C_{p} R_{L}+1\right)^{2} \cos ^{-1}\left(\frac{4 f C_{p} R_{L}-1}{4 f C_{p} R_{L}+1}\right)}
\end{aligned}
$$




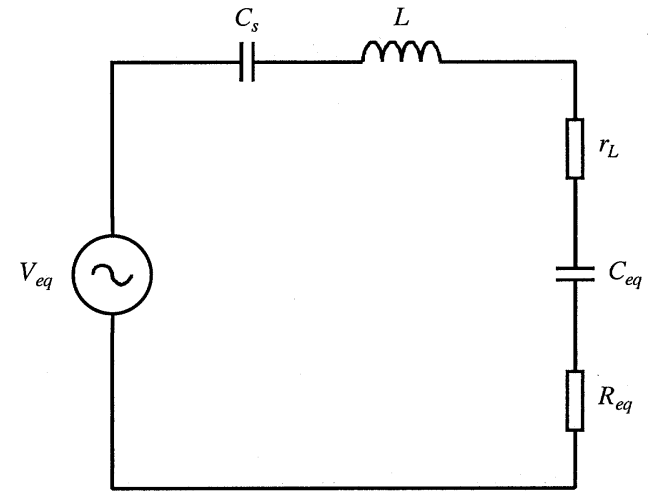

Fig. 3. Equivalent circuit of an LCC voltage output converter.

(29), shown at the bottom of the previous page. The converter is analyzed by assuming the action of the rectifier is to clamp the parallel capacitor voltage (to $\pm v_{o}$ ) when the rectifier is conducting. The parallel capacitor voltage can then be described by (30), shown at the bottom of the page, where $V_{b}=V_{\text {out }}+2 V_{d}$ and $I_{i n}$ is the input current to the converter, $f$ is the switching frequency, and $\theta$ is the parallel capacitor charging angle.

The duty-cycle for mode $1\left(d_{1}\right)$ is found by assuming the output voltage is ripple free. Hence, when the bridge rectifier is conducting the current flowing through $C_{p}$ is negligible. The current flowing through the rectifier is then equal to the inductor current, i.e. $i_{R}=i_{L}$. The time $t=t_{1}$ is defined as the time at which $i_{R}=0$, and can be derived by finding the impedance of the equivalent circuit in Fig. 3

$$
X=\frac{s^{2} L C_{t o t}+s C_{t o t} R_{e q}+1}{s C_{t o t}}
$$

where $C_{t o t}=C_{s} C_{e q} /\left(C_{s}+C_{e q}\right) \cdot d_{1}$ is obtained from the polar representation of (31) and is normalized by dividing by $2 \pi$

$$
d_{1}=\frac{1}{2 \pi} \tan ^{-1}\left(\frac{\omega^{2} L C_{t o t}-1}{\omega C_{t o t} R_{e q}}\right) .
$$

The duty-cycle for mode $2\left(d_{2}\right)$ is found from the integral

$$
v_{C p}=v_{C p}\left(t_{1}\right)+\frac{1}{C_{p}} \int_{t_{1}}^{t_{2}} I_{i n} \sin (2 \pi f t) d t
$$

which describes the parallel capacitor voltage when $i_{R}=0$. By noting that at time $t=t_{1}, v_{C p}=-V_{b}$ and at time $t=t_{2}$, $v_{C p}=V_{b}$, (33) can be solved for $t_{2}$. The duty-cycle $d_{2}$ is then given by

$$
d_{2}=\frac{t_{2}}{T}=\frac{1}{2 \pi} \cos ^{-1}\left(1-\frac{4 \pi V_{b} f C_{p}}{I_{i n}}\right) .
$$

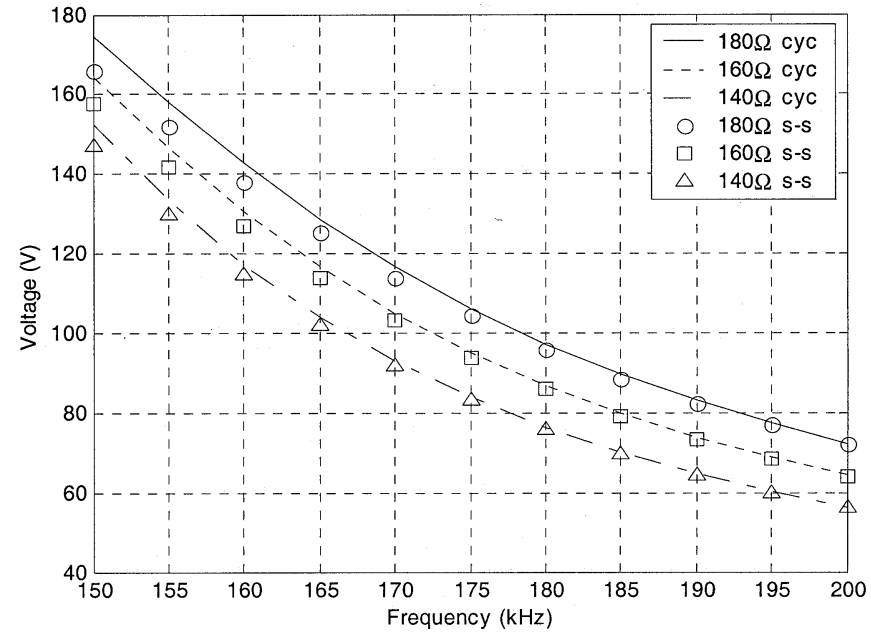

Fig. 4. Comparison between output voltage predicted by the state-space model (s-s) and cyclic averaging (cyc).

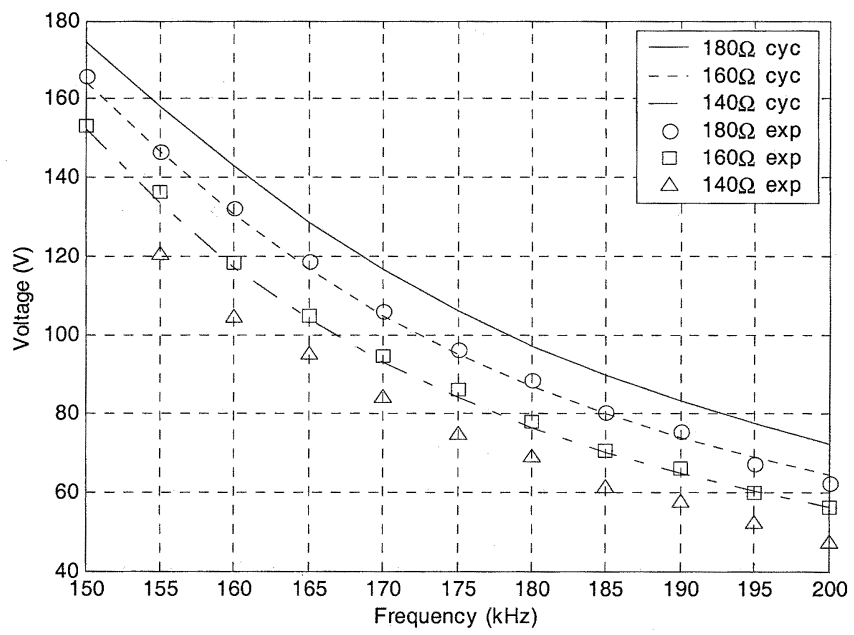

Fig. 5. Comparison between output voltage predicted by cyclic averaging (cyc) and measured on experimental converter (exp).

The remaining duty-cycles are found by symmetry

$$
\begin{aligned}
& d_{3}=0.5-d_{1}-d_{2} \\
& d_{4}=d_{1} \\
& d_{5}=d_{2} \\
& d_{6}=d_{3} .
\end{aligned}
$$

Finally, from the state-space descriptions of each mode in a cycle, (26)-(28), and the duty-cycles which determine the time duration of each mode, it is possible to describe the converter in the cyclic mode from (8), (9), (13), and (14).

$$
v_{C p}=\left\{\begin{array}{cc}
-V_{b}+\frac{I_{i n}}{2 \pi f C_{p}}(1-\cos (\theta)) & \theta=0 \ldots \cos ^{-1}\left(1-\frac{4 \pi V_{b} f C_{p}}{I_{i n}}\right) \\
V_{b} & \theta=\cos ^{-1}\left(1-\frac{4 \pi V_{b} f C_{p}}{I_{i n}}\right) \ldots \pi \\
V_{b}-\frac{I_{i n}}{2 \pi f C_{p}}(1+\cos (\theta)) & \theta=\pi \ldots \pi+\cos ^{-1}\left(1-\frac{4 \pi V_{b} f C_{p}}{I_{i n}}\right) \\
-V_{b} & \theta=\pi+\cos ^{-1}\left(1-\frac{4 \pi V_{b} f C_{p}}{I_{i n}}\right) \ldots 2 \pi
\end{array}\right.
$$




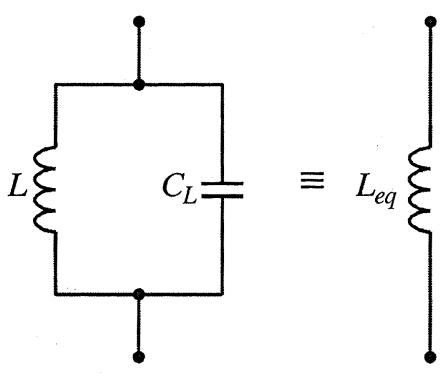

(a)

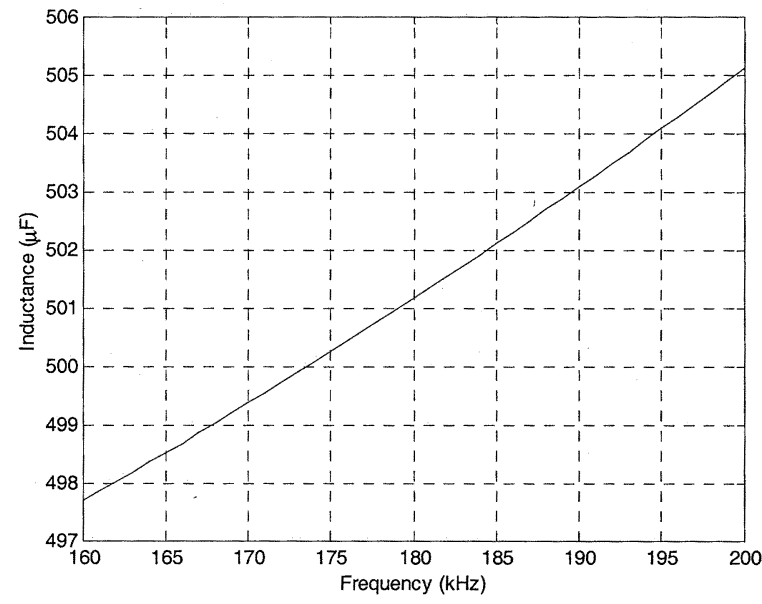

(b)

Fig. 6. (a) Equivalent circuit of resonant inductor and (b) $L_{e q}$ versus frequency.

\section{Simulation AND EXPERIMENTAL Results}

The cyclic averaging methodology is now employed to rapidly predict the steady-state output voltage of a converter model, and the accuracy of the results is assessed. A benchmark converter has been designed to provide an output voltage of $150 \mathrm{~V}$ at a nominal operating frequency of $160 \mathrm{kHz}$, with an input voltage of $350 \mathrm{~V}$. The resulting circuit component values are $L=485 \mu \mathrm{H}$ (measured), $C_{s}=3 \mathrm{nF}, C_{p}=680 \mathrm{pF}$, $C_{f}=47 \mu \mathrm{F}$ and $R_{L}=180$.

Fig. 4 compares the output voltage obtained by simulating the state-space model to steady-state $(20 \mathrm{~ms})$ to that predicted by cyclic-averaging, for a variety of loads. As will be evident, the results compare favorably, although the data obtained using cyclic averaging took only $1 / 10000$ th of the execution time of the state-space model. (However, of course, only steady-state results are obtained).

In order to determine the accuracy of the proposed cyclic-averaging methodology for determining the steady-state operation of a practical converter, a half-bridge LCC voltage output converter is having the component values given previously, is considered. Fig. 5 compares the output voltage of the experimental converter with predictions from cyclic averaging, for a range of loads.

The discrepancy between the cyclic averaging predictions and the experimental results is indicative of a shift in resonant frequency, and is attributed to the self-capacitance of the resonant inductor $L$. The stray-capacitance of the resonant inductor was measured as $\approx 52 \mathrm{pF}$ at resonance. To incorporate the effects of the self-capacitance, the parallel combination of $L$ and its parasitic capacitance $C_{L}$ is modeled by an equivalent, frequency dependent, inductance $L_{e q}$ (36), as illustrated in Fig. 6.

The value of the equivalent inductance, $L_{e q}$, is obtained from the equivalent impedance of the parallel $L$ and $C_{L}$ combination

$$
Z_{e q}=\frac{j \omega L}{1-\omega^{2} L C_{L}}=j \omega L_{e q} \text { where } L_{e q}=\frac{L}{1-\omega^{2} L C_{L}} .
$$

The predicted output voltage of the converter the after the modified value of the inductance is incorporated into the cyclic

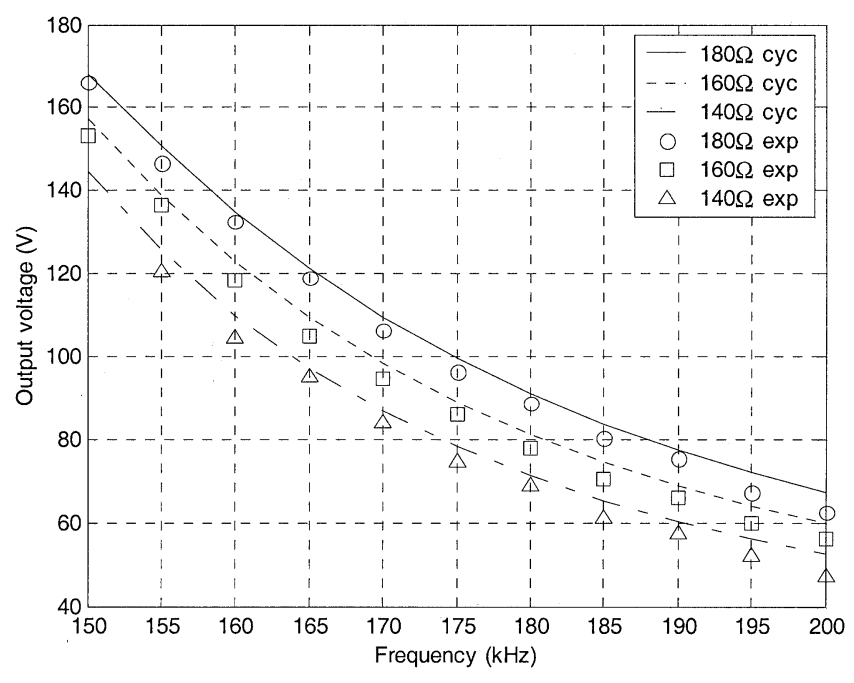

Fig. 7. Comparison between output voltage predicted by compensated cyclic averaging (cyc) and measured on the experimental converter (exp).

averaging algorithm shown in Fig. 7; which again includes the measurements for comparison purposes. The improved correlation between the theoretical predictions and the experimental data is self-evident.

\section{COMPONENT STRESSES}

During the converter design process, it is necessary to consider the peak voltage and current stresses which are imposed on the resonant components, with a view to establishing the required component ratings. It is now shown that cyclic-averaging provides a convenient, accurate, and computationally efficient method for addressing this issue.

Referring once again to Fig. 2, it can be seen that the peak voltage across the series capacitor, $C_{s}$, occurs at the instant of time when the current that flows through the resonant inductor passes through zero. The time at which $i_{L}=0\left(t_{C s_{-} \max }\right)$ is obtained from $d_{1}$, (32). Substitution of $t_{C s \_ \text {max }}$ into (9) provides the following expression for $v_{C s_{-} \max }$ :

$$
v_{\text {cs_max }}=\left[\begin{array}{lllll}
0 & -1 & 0 & 0 & 0
\end{array}\right] e^{\hat{A}_{1} d_{1} T} \hat{x}_{p e r}\left(t_{0}\right) .
$$




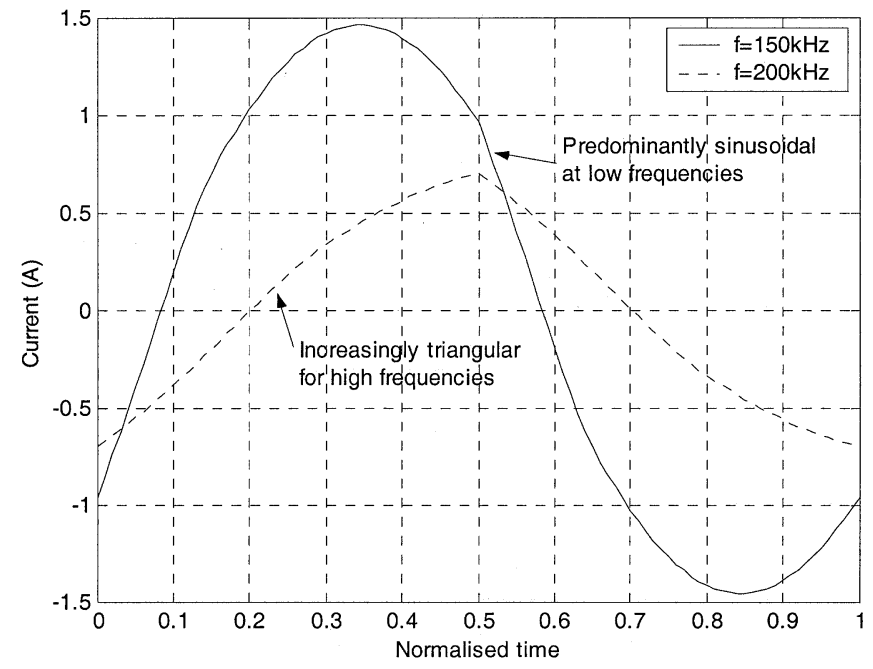

Fig. 8. Change in inductor current waveform with increasing frequency.

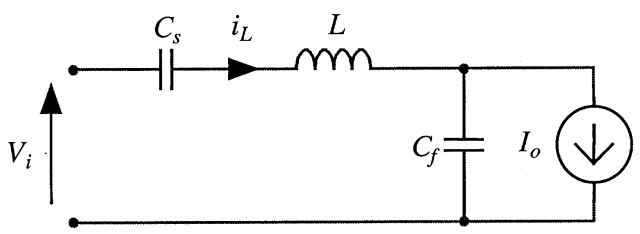

Fig. 9. Equivalent circuit of LCC voltage-output converter operating in mode 3.

Fundamental mode analysis (FMA) techniques can be used to predict the time at which $i_{L}$ reaches its maximum value. However, as the switching frequency is increased, the inductor current waveforms become less sinusoidal and increasingly triangular, see Fig. 8; and FMA thus becomes less accurate.

In order to improve the accuracy of the predictions, therefore, the inductor current is modeled by considering the 'equivalent circuit' shown in Fig. 9. This is justified since the majority of the inductor current flows through the rectifier, and hence, $C_{p}$ has a negligible effect on the current. Also, if the filter capacitor, $C_{f}$, is assumed to be sufficiently large that there is zero output voltage ripple, the load resistance can be replaced by an equivalent current sink of value $I_{o}$, where $I_{o}=\left(v_{O \_} a v / R_{L}\right)$ and $v_{O_{-} a v}$ is the output voltage $v_{o}$ averaged over one cycle in steady-state, (14).

The dynamical equations governing the operation of the converter in mode 3 are then

$$
\begin{aligned}
\dot{i}_{L} & =\frac{V_{i}-v_{c s}-v_{c f}}{L} \\
\dot{v}_{C s} & =\frac{i_{L}}{C_{S}} \\
\dot{v}_{C f} & =\frac{i_{L}-I_{O}}{C_{f}} .
\end{aligned}
$$

To simplify the mathematics, both of the capacitor voltages can be represented by one state-variable, by summing their contribution to produce a combined capacitor voltage $v_{c}$ [5]. Represented in state-space form, the voltage combination produces the new system description (39)

$$
\left[\begin{array}{l}
\dot{v}_{c} \\
\dot{i}_{L}
\end{array}\right]=\left[\begin{array}{cc}
0 & \frac{1}{C_{T}} \\
-\frac{1}{L} & 0
\end{array}\right]\left[\begin{array}{l}
v_{c} \\
i_{L}
\end{array}\right]+\left[\begin{array}{cc}
0 & -\frac{1}{C_{f}} \\
\frac{1}{L} & 0
\end{array}\right]\left[\begin{array}{c}
V_{i} \\
I_{O}
\end{array}\right]
$$

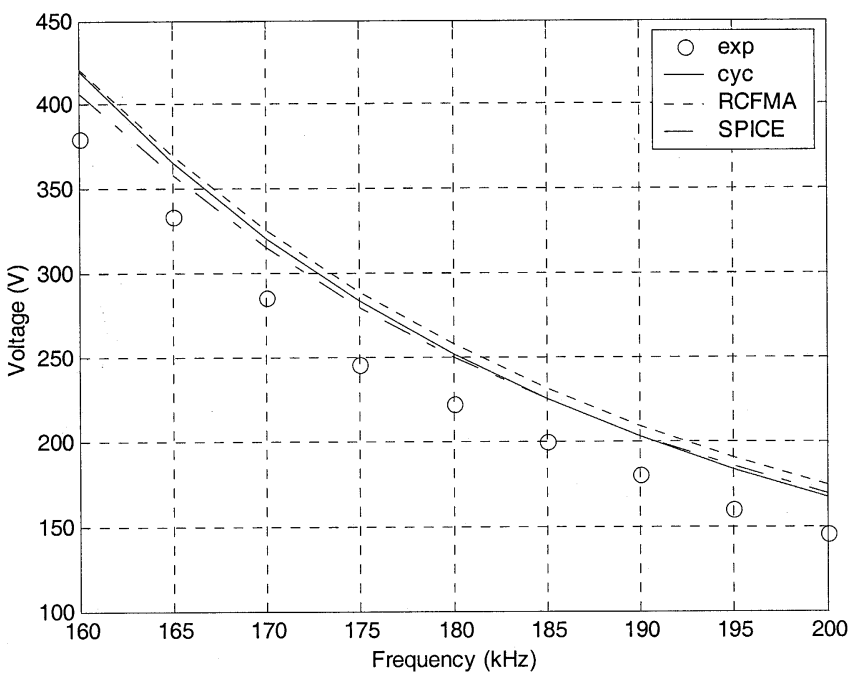

(a)

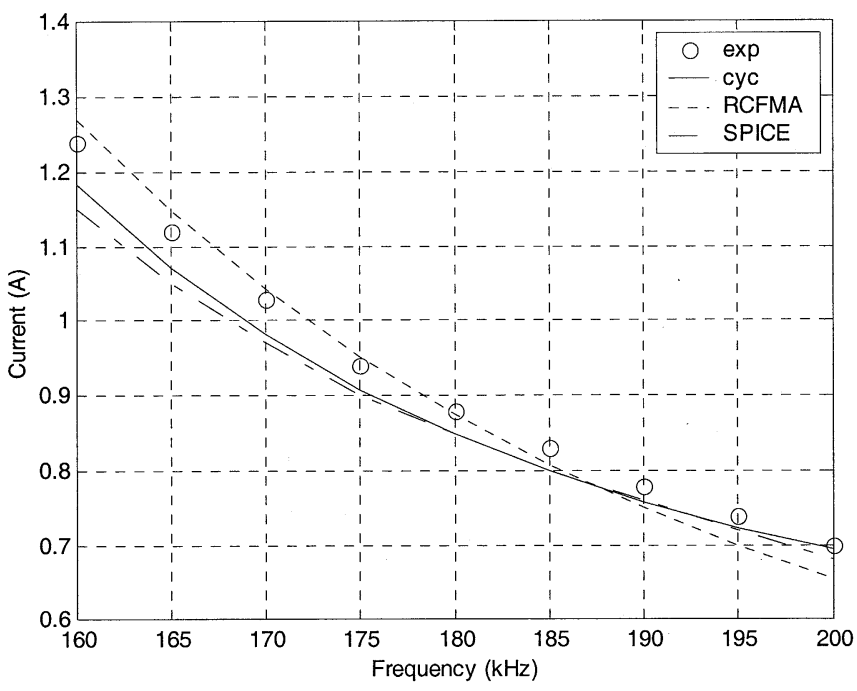

(b)

Fig. 10. (a) Series capacitor voltage and (b) inductor current stresses for $180 \Omega$ load.

where $v_{c}=v_{C s}+v_{C f}, C_{T}=C_{s} C_{f} /\left(C_{s}+C_{f}\right)$.

The solution for $i_{L(t)}$ can be shown to be

$$
\begin{aligned}
i_{L}(t)= & \frac{I_{o} C_{T}}{C_{f}}+\sqrt{L C_{T}\left(\frac{V_{i}-v_{c}\left(t_{2}\right)}{L}-\frac{I_{o} C_{T}}{C_{f}}\right)^{2}+i_{L}\left(t_{2}\right)^{2} \times} \\
& \sin \left(\frac{1}{\sqrt{L C_{T}}} t+\tan ^{-1}\right. \\
& \left.\times\left[\frac{i_{L}\left(t_{2}\right)}{\sqrt{L C_{T}}\left(\frac{V_{i}-v_{c}\left(t_{2}\right)}{L}-\frac{I_{o} C_{T}}{C_{f}}\right)}\right]\right) \cdot(40)
\end{aligned}
$$

To obtain the time at which the maximum inductor current flows, (40) is differentiated, equated to zero, and solved for $t$, giving

$t_{i L \_\max }=\sqrt{L C_{T}}\left[\frac{\pi}{2}-\tan ^{-1}\left(\frac{i_{L}\left(t_{2}\right)}{\sqrt{L C_{T}}\left(\frac{V_{i}-v_{c}\left(t_{2}\right)}{L}-\frac{I_{o} C_{T}}{C_{f}}\right)}\right)\right]$. 


$$
t_{i L \_ \text {max }}=\left\{\begin{array}{cc}
\sqrt{L C_{T}}\left[\frac{\pi}{2}-\tan ^{-1}\left(\frac{i_{L}\left(t_{2}\right)}{\sqrt{L C_{T}}\left(\frac{V_{i}-v_{C}\left(t_{2}\right)}{L}-\frac{I_{o} C_{T}}{C_{f}}\right)}\right)\right] & t_{i L_{-} \max }<d_{3} T \\
d_{3} T & t_{i L_{-} \max } \geq d_{3} T
\end{array}\right.
$$

When operating above resonance the inductor current lags the input voltage. As shown in Fig. 8, at high switching frequencies (with respect to the resonant frequency) the inductor current does not have sufficient time to reach its 'natural' peak value, and the waveform becomes increasingly triangular (with increasing frequency). Equation (41) is, therefore, modified to account for this effect by limiting the maximum value at which $t_{i L_{-} \max }$ can occur, resulting in (42), shown at the top of the page. From (42) it is readily shown that the maximum inductor current is then given by

$$
i_{L_{-} \max }=\left\{\begin{array}{ccc}
{\left[\begin{array}{ccccc}
0 & 0 & 1 & 0 & 0
\end{array}\right] e^{\hat{A}_{3} t_{i L \_m a x}}} & \\
& \times e^{\hat{A}_{2} d_{2} T} e^{\hat{A}_{1} d_{1} T} \hat{x}_{p e r}\left(t_{0}\right) & t_{i L \_ \text {max }}<d_{3} T \\
{\left[\begin{array}{ccccc}
0 & 0 & 1 & 0 & 0
\end{array}\right] e^{\hat{A}_{3} d_{3} T}} & \\
& \times e^{\hat{A}_{2} d_{2} T} e^{\hat{A}_{1} d_{1} T} \hat{x}_{p e r}\left(t_{0}\right) & t_{i L \_ \text {max }} \geq d_{3} T
\end{array}\right.
$$

The steady-state stresses on the resonant converter components then follow from (37) and (43). Fig. 10 compares the measured of stresses on the experimental converter with theoretical predictions, all predictions utilising the modified inductance value, (36). For completeness, results obtained from both Spice simulations and RCFMA [4] are also included.

Again, it will be seen that good correlation exists between predictions generated using cyclic averaging and those deduced from Spice. However, the execution time to obtain the data by cyclic-averaging was approximately 1/10000 times that which was required for the Spice simulations.

\section{CONCLUSION}

The paper has described the application of a cyclic-averaging based technique to facilitate the rapid analysis and design of high order resonant power converters. It has been shown that cyclic-averaging is an appropriate method for obtaining steady-state performance predictions of the current and voltage waveforms, as evidenced by comparisons with state-space and Spice based simulations and measurements made on an experimental converter. It has also been shown that the method takes only $\sim 1 / 10000$ th of the computation time of Spice. Moreover, a comparison of results has shown that cyclic averaging provides reliable predictions of voltage and current stresses on the resonant circuit components.

\section{REFERENCES}

[1] H. R. Visser and P. P. J. van den Bosch, "Modeling of periodically switched networks," in Proc. 22nd IEEE Power Electron. Spec. Conf. (PESC'91), Boston, MA, 1991, pp. 67-73.

[2] J. Sun and H. Grotstollen, "Symbolic analysis of switching power converters based on a general averaging method," in Proc. 27th IEEE Power Electron. Spec. Conf. (PESC'96), Baveno, Italy, 1996, pp. 543-549.

[3] M. P. Foster, H. I. Sewell, C. M. Bingham, and D. A. Stone, "State-variable modeling of LCC voltage output converters," IEE Electron. Lett., vol. 17, pp. 1065-1066, 2001.
[4] J. G. Hayes and M. G. Egan, "Rectifier-compensated fundamental mode approximation analysis of the series-parallel LCLC family of resonant converters with capacitative output filter and voltage-source load," in Proc. 30th IEEE Power Electron. Spec. Conf. (PESC'99), Charleston, SC, 1999, pp. 1030-1036.

[5] I. Batarseh, R. Liu, C. Q. Lee, and A. K. Upadhyay, "Theoretical and experimental studies of the LCC-Type parallel resonant converter," IEEE Trans. Power Electron., vol. 5, pp. 140-150, Apr. 1990.

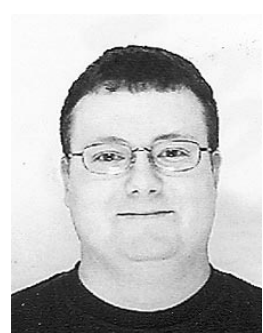

Martin P. Foster received the B.Eng. degree in electronic and electrical engineering and the M.Sc. degree in control systems from the University of Sheffield, U.K., in 1998 and 2000, respectively, where he is currently pursuing the $\mathrm{Ph} . \mathrm{D}$. degree.

His research interests include the modeling and control of switching power converters with particular emphasis on high-efficiency resonant supplies.

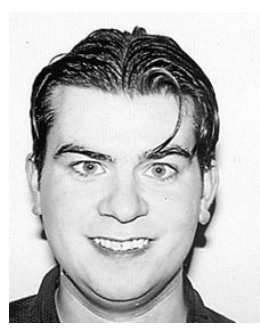

H. Isaac Sewell received the M.Eng degree in electronic and electrical engineering and the Ph.D. degree from the University of Sheffield, U.K., in 1996 and 2002 , respectively.

Since 2000, he has worked in industry as a Design Engineer at Inductelec, Ltd, Sheffield, and as a Research Associate in the Department of Electronic and Electrical Engineering, University of Sheffield, where his current research interests include induction heating, mains supply power-factor correction, and the analysis and design of resonant power converters.

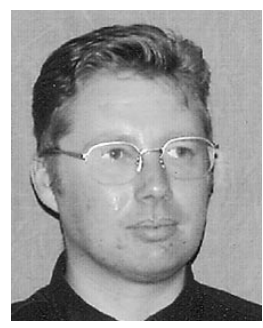

Chris M. Bingham (M'90) received the B.Eng degree in electronic systems and control engineering from Sheffield City Polytechnic, U.K., in 1989, the M.Sc. degree in control systems engineering from the University of Sheffield, U.K., in 1990, and the Ph.D. degree in control systems to accommodate nonlinear dynamic effects in aerospace flight-surface actuators from Cranfield University, U.K., in 1994.

He subsequently took up a research position at the University of Sheffield. Since 1998, he has been a Lecturer in the Department of Electronic and Electrical Engineering, University of Sheffield. His current research interests include traction control/anti-lock braking systems for electric vehicles, electro-mechanical actuation of flight control surfaces, control of active magnetic bearings for high-speed machines, sensorless control of brushless machines, and the high speed modeling, analysis and design of resonant power converters.

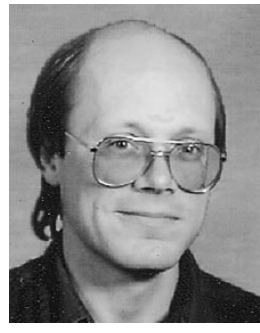

David A. Stone received the B.Eng. degree in electronic engineering from the University of Sheffield, U.K., in 1984 and the Ph.D. degree from Liverpool University, U.K., in 1989.

He then returned to the University of Sheffield as a member of academic staff specializing in power electronics and machine drive systems. His current research interests are in resonant power converters, hybrid-electric vehicles, battery charging, EMC and novel lamp ballasts for low-pressure fluorescent lamps. 
Dirk Hente received the Dipl.Ing. degree in electrical engineering from the Fachhochschule Bielefeld, Germany, in 1979, the Dipl.Ing. degree in electrical engineering from the Technische Universität Berlin, Germany, in 1982, and the Dr.Ing. degree in bifurcation theory for nonlinear electrical networks from the Universität Gesamthochschule Duisburg, Germany, in 1986.

In 1986, he joined Philips Research Laboratories, Aachen, Germany, as a Research Scientist. His current research interests include resonant converter design, circuit simulation, and electromagnetic field theory.

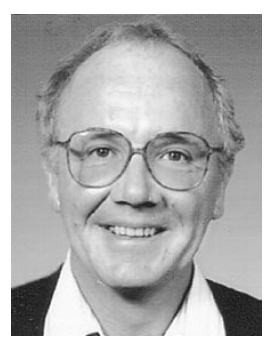

Dave Howe received the B.Tech. and M.Sc. degrees from the University of Bradford, U.K., in 1966 and 1967, respectively, and the Ph.D. degree from the University of Southampton, U.K., in 1974, all in electrical power engineering.

$\mathrm{He}$ has held academic posts at Brunel and Southampton Universities, and spent a period in industry with NEI Parson, Ltd., working on electromagnetic problems related to turbo-generators. He is currently Professor of Electrical Engineering at the University of Sheffield, U.K., where he heads the Electrical Machines and Drives Research Group. His research activities span all facets of controlled electrical drive systems, with particular emphasis on permanent magnet excited machines.

Dr. Howe is a Chartered Engineer and a Fellow of the IEE, U.K. 